\title{
Development and Validation of RP-HPLC Method for Simultaneous Determination of Curcumin and Quercetin in Extracts, Marketed Formulations, and Self-Nanoemulsifying Drug Delivery System
}

Rubiya Khursheed ${ }^{1}$, Sachin Kumar Singh ${ }^{1 *}$, Bhupinder Kapoor ${ }^{1}$, Monica Gulati', Sheetu Wadhwa ${ }^{1}$, Saurabh Gupta ${ }^{2}$, Parteek Prasher $^{3}$, Deepak Kumar ${ }^{4}$, Kamal Dua ${ }^{5}$, L. M. Sharath Kumar ${ }^{6}$, U. V. Babu ${ }^{6}$, Manish Sharma${ }^{7}$, Hardik K. Soni $^{7}$, and Vijay Kumar ${ }^{8}$

\begin{abstract}
A simple high-performance liquid chromatography (HPLC) method has been established and validated for the simultaneous determination of curcumin and quercetin. The method was applied for their quantification in marketed formulations, extracts, and developed self-nano emulsifying drug delivery system (SNEDDS) formulation. The mobile phase used for the chromatographic runs comprised of glacial acetic acid (GAA) (2\% v/v) and acetonitrile $(A C N)$ in a gradient elution. The method has been validated according to International Conference of Harmonization (ICH) Q2 (R1) guidelines with respect to specificity, system suitability, accuracy, precision, and robustness. The developed method was further used for the determination of curcumin and quercetin in different herbal extracts. The method was linear in the concentration range of $50-250 \mathrm{ng} \mathrm{mL}^{-1}$ for curcumin and $800-$ $1600 \mathrm{ng} \mathrm{mL}^{-1}$ for quercetin, respectively. The limit of detection (LOD) and limit of quantification (LOQ) were 11.76 and $35.6 \mathrm{ng} \mathrm{mL}^{-1}$ for curcumin, respectively, while LOD and LOQ for quercetin were 32.94 and $99.76 \mathrm{ng} /$ $\mathrm{mL}^{-1}$, respectively.
\end{abstract}

Keywords: HPLC; SNEDDS; curcumin; quercetin

Pages: $43-51$

\section{Introduction}

Curcumin and quercetin are the two important flavonoids that have gained consideration in the last 30 years for their antidiabetic, antioxidant, and anti-inflammatory effects. Several clinical and preclinical studies have shown the potential effect of these two flavonoids in the treatment of different diseases such as arthritis, ${ }^{1}$ diabetes, ${ }^{2,3}$ cancer, ${ }^{3}$ cardiovascular diseases, ${ }^{4,5}$ and bacterial infections. ${ }^{6,7}$ Numerous actions of curcumin are linked to its various activities on the glycogen synthase kinase 3-beta (GSK-3 $\beta$ ) pathway. ${ }^{8}$ Mechanisms of action of quercetin are pleiotropic and involve the inhibition of intestinal glucose absorption, insulin secretory, and insulin-sensitizing activities as well as improved glucose utilization in peripheral tissues. ${ }^{9,10}$ Moreover, both the drugs act on different pathways that are involved in diseases such as diabetes, cancer, neurodegenerative

${ }^{1}$ School of Pharmaceutical Sciences, Lovely Professional University, Phagwara 144411, Punjab, India; ${ }^{2}$ Chitkara College of Pharmacy, Chitkara University, Chandigarh 140401, Punjab, India; ${ }^{3}$ Department of Chemistry, University of Petroleum \& Energy Studies, Energy Acres, Dehradun 248007 , Uttarakhand, India; ${ }^{4}$ Department of Pharmaceutical Chemistry, School of Pharmaceutical Sciences, Shoolini University, Solan 173229, Himachal Pradesh, India; ${ }^{5}$ Discipline of Pharmacy, Graduate School of Health, University of Technology Sydney, Sydney 2007, New South Wales, Australia; ${ }^{6}$ Phytochemistry Department, The Himalaya Drug Company, Makali, Bengaluru 562162, Karnataka, India; ${ }^{7}$ Vasu Research Centre, GIDC, Vadodara 390010, Gujarat, India; ${ }^{8}$ Department of Biotechnology, School of Bioengineering and Biosciences, Faculty of Technology and Sciences, Lovely Professional University, Phagwara 144411, Punjab, India

*Address correspondence to: Sachin Kumar Singh, School of Pharmaceutical Sciences, Lovely Professional University, Phagwara 144411, Punjab, India, Email: Singhsachin23@gmail.com; sachin.16030@lpu.co.in

(C) Rubiya Khursheed, Sachin Kumar Singh, Bhupinder Kapoor, Monica Gulati, Sheetu Wadhwa, Saurabh Gupta, Parteek Prasher, Deepak Kumar, Kamal Dua, L. M. Sharath Kumar, U. V. Babu, Manish Sharma, Hardik K. Soni, and Vijay Kumar 2021; Published by Mary Ann Liebert, Inc. This Open Access article is distributed under the terms of the Creative Commons License (http://creativecommons.org/licenses/by/4.0), which permits unrestricted use, distribution, and reproduction in any medium, provided the original work is properly cited. 
diseases, and inflammation. These pathways include regulation of adenosine $5^{\prime}$ monophosphate-activated protein kinase (AMPK), peroxisome proliferator-activated receptor gamma (PPAR- $\gamma$ ), phosphoinositide 3-kinaseprotein kinase B, c-Jun N-terminal kinase (JNK), and Janus tyrosine kinase-signal transducer and activator of transcription (JAK-STAT) pathways. ${ }^{11-16}$ Many highperformance liquid chromatography (HPLC) methods either in pharmaceutical products or in biological samples are reported in the literature for the determination of curcumin alone,${ }^{17,18}$ quercetin alone, ${ }^{19,20}$ curcumin with other drugs, ${ }^{21,22}$ and quercetin with other drugs. ${ }^{23}$ Since, both curcumin and quercetin have been used in many polyherbal formulations as a health supplement for the management of obesity, ${ }^{24,25}$ improving immunity, ${ }^{26}$ adenomatous polyposis, ${ }^{27}$ and so on. The combination of both these drugs is available in the market as Vimerson Health in combination with bromelain. ${ }^{28}$ Hence, there is a need to develop a simultaneous method for quercetin and curcumin. Very few methods for simultaneous estimation of curcumin and quercetin have been reported. ${ }^{29-31}$ Advanced methods such as ultraperformance liquid chromatography-mass spectrometry (UPLC MS-MS) have been used for simultaneous determination of curcumin and quercetin in rat plasmas. However, this method is complex and requires costly instrumentation. ${ }^{29}$ Moreover, the HPLC method has been reported for the simultaneous determination of curcumin and quercetin. Thus, the limitation of this method is a long run time (20 minutes). ${ }^{32}$

This current study is aimed to develop and validate a simple, economic, rapid, sensitive, and gradient HPLC method for the simultaneous determination of curcumin and quercetin. The developed method has been successfully applied to determine the concentrations of curcumin and quercetin in various plant extracts, extract-based marketed formulations, and in-house formulated selfnano emulsifying drug delivery systems (SNEDDSs).

\section{Experimental}

Materials, Reagents, and Pharmaceutical Products

Curcumin was purchased from HiMedia (Mumbai, India). Quercetin hydrate was purchased from Molychem (Mumbai, India). HPLC grade ACN and GAA were purchased from Rankem (Maharashtra, India). Propylene glycol (PG), Tween 20, 80, polyethylene glycol (PEG) 200, 400, and 600, Span 80, sesame oil, soyabean oil, groundnut oil, cottonseed oil, almond oil, eucalyptus oil, mustard oil, olive oil, and castor oil were purchased from Central Drug House (CDH) (New Delhi, India). Labrafac ${ }^{\circledR} \quad$ PG, Labrafil ${ }^{\circledR}$ M1944CS, Lauroglycol ${ }^{\circledR}$
FCC, Labrasol $®$, and Transcutol $\AA$ P were received as a gift sample from M/s Gattefosse (Mumbai, India). Capmul ${ }^{\circledR}$ MCM was obtained as a gift sample from M/s Abitec Corp. (Mumbai, India). Turmeric formula capsules were purchased from Organic India (Lucknow, India). Haridra capsules were purchased from Himalaya herbal healthcare (Bangalore, India). Curpin capsules were obtained from Vaibhav Herbal (Amritsar, India). Haldi immunity enhancer capsules were purchased from Ayush Pharmaceuticals (Kangra, India). Haridra Khanda extract was purchased from Dabur (New Delhi, India). Haridra Khand powder extract was purchased from Wilson Drugs and Pharmaceuticals (Himachal Pradesh, India). Different quercetin extracts (onion, pudina, ajwain, amla, chebula, ginger, green tea, nutmeg, pepper, pomegranate, and tulsi) were gifted from Himalaya herbals (Bangalore, India).

\section{Spectral Analysis}

Each drug sample (curcumin and quercetin) and their mixture $\left(10 \mu \mathrm{g} \mathrm{mL}^{-1}\right.$ each) were prepared in ACN, and it was scanned for the determination of absorption maxima (UV-1800, Shimadzu, Japan) in the range of 200-600 nm against ACN as blank. In addition to that the solution of their physical mixture was also scanned in the same range.

\section{Analytical Method Development}

The analytical method was developed by using an HPLC system (Shimadzu LC-20AD Prominence, Japan) with a photodiode array detector (SPD-M20A) and a Rheodyne injector (7725i) equipped with an LC20AD pump. Statistical acquisition, recording, and chromatographic integration were achieved using LabSolutions version 5.82. Analysis and separation have been done on the C18 column with a length of $250 \mathrm{~mm}$, an internal diameter of $4.6 \mathrm{~mm}$, and a bead size of $5 \mu \mathrm{m}$. Gradient elution was carried out using 2\% GAA and ACN as the mobile phase. The mobile phase consisted of GAA $(2 \% \mathrm{v} / \mathrm{v})$ and ACN in a gradient elution. The flow rate was set at

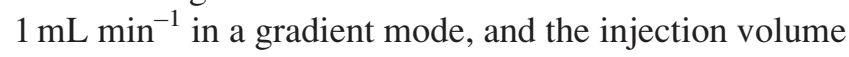
was set at $20 \mu \mathrm{L}$ for all samples.

Preparation of the acetic acid solution. In the method development, a $2 \% \mathrm{v} / \mathrm{v}$ solution of GAA was used as one of the components of the mobile phase. It was prepared by adding $2 \mathrm{~mL}$ acetic acid in $1000 \mathrm{~mL}$ distilled water, and the final $\mathrm{pH}$ was then checked by using a $\mathrm{pH}$ meter. The solution was degassed using vacuum filtration $(0.45 \mu \mathrm{m}$ nylon membrane filter) and ultrasonication. Ultrasonication was carried out for 10 minutes using a Labman-ROHS (China) sonicator. 
Preparation of the stock solution for curcumin and quercetin. Curcumin and quercetin $(10 \mathrm{mg}$ each) were weighed and transferred into a 100 -mL volumetric flask. To this, $20 \mathrm{~mL}$ of ACN was added to dissolve the drugs. Then, the final volume was adjusted to $100 \mathrm{~mL}$ using $\mathrm{ACN}$. This was labeled as solution A. The concentration of both curcumin and quercetin was $100 \mu \mathrm{g} \mathrm{mL} \mathrm{m}^{-1}$ in solution A.

Preparation of the working solution. One milliliter aliquot from solution $\mathrm{A}$ was withdrawn and transferred into a $10-\mathrm{mL}$ volumetric flask (labeled as solution B), and the final volume was made up to $10 \mathrm{~mL}$ with $\mathrm{ACN}$ to give a working solution of curcumin $\left(10 \mu \mathrm{g} \mathrm{mL}^{-1}\right)$ and quercetin $\left(10 \mu \mathrm{g} \mathrm{mL}^{-1}\right)$. From solution $\mathrm{B}$, an aliquot of $1 \mathrm{~mL}$ was withdrawn and transferred to another flask (labeled as solution $\mathrm{C}$ ), and the final volume was made up to $10 \mathrm{~mL}$ to give a working solution of $1 \mu \mathrm{g} \mathrm{mL} \mathrm{m}^{-1}$ for both the drugs.

Preparation of sample dilutions for curcumin and quercetin. Ten volumetric flasks with a capacity of $10 \mathrm{~mL}$ were taken and labeled as D1, D2, D3, D4, D5, D6, D7, D8, D9, and D10. The flasks D1-D5 and D6D10 were used to prepare dilutions of curcumin and quercetin, respectively. Accurately measured volumes of $0.5,1,1.5,2$, and $2.5 \mathrm{~mL}$ from flask $\mathrm{C}$ were transferred in flasks D1, D2, D3, D4, and D5, respectively. The final volume of each flask was made up to $10 \mathrm{~mL}$ to produce final concentrations of 50, 100, 150, 200, and $250 \mathrm{ng} \mathrm{mL}^{-1}$ for curcumin. To prepare serial dilutions for quercetin, accurately measured volumes of $0.8,1$, $1.2,1.4$, and $1.6 \mathrm{~mL}$ from flask $\mathrm{B}$ were transferred to flasks D6, D7, D8, D9, and D10. The volume in each flask was made up to $10 \mathrm{~mL}$ to produce final concentrations of $800,1000,1200,1400$, and $1600 \mathrm{ng} \mathrm{mL}^{-1}$ for quercetin. The samples were further analyzed on HPLC for different parameters of validation.

Method development and optimization. Due to the significant variance in the chemical and physical properties of curcumin and quercetin, various mobile phases and elution methods were initially tried in order to have both eluents on the same chromatogram. The mobile phase composition and the gradient elution used in the optimized method have been decided based on sensitivity and selectivity as well as suitable chromatographic parameters of the developed peaks in terms of peak shape, peak purity, peak sharpness, tailing factor, and resolution between the two peaks. The mobile phase was used as a solvent for all samples to confirm least noise and to eradicate any undesirable solvent peaks. Various trials were carried out in order to find better chromatographic conditions. The concentration of curcumin and quercetin for developing the analytical method was $10 \mu \mathrm{g} \mathrm{m}^{-1}$.

In trial 1 , the isocratic mode of elution was carried out using ACN and formic acid $(0.1 \% \mathrm{v} / \mathrm{v})$ in the ratio of $60: 40 \mathrm{v} / \mathrm{v}$ as the mobile phase. In trial 2, gradient elution in $\mathrm{ACN}$ and water was carried out for 20 minutes, wherein the ratio of $\mathrm{ACN}$ and water was kept at 35:65 v/ $\mathrm{v}$ for 4 minutes followed by $35-80 \mathrm{v} / \mathrm{v}: 65-20 \mathrm{v} / \mathrm{v}$ between 4 and 8 minutes and $80 \mathrm{v} / \mathrm{v}: 20 \mathrm{v} / \mathrm{v}$ between 8 and 20 minutes. In trial 3 , isocratic elution was carried out using $\mathrm{ACN}$ and orthophosphoric acid (OPA, 0.1\% v/v) in the ratio of $50: 50 \mathrm{v} / \mathrm{v}$ as the mobile phase. In trial 4 , isocratic elution was carried using CAN and GAA $(2 \%$ $\mathrm{v} / \mathrm{v}$ ) in the ratio of 60:40 as the mobile phase. In trial 5, gradient elution in ACN and GAA (2\% v/v) was carried out for 15 minutes, wherein the ratio of $\mathrm{ACN}$ and GAA was varied from 40 to $60 \mathrm{v} / \mathrm{v}$ ACN:60-40 GAA v/v from 0 to 5 minutes followed by $60-80 \mathrm{v} / \mathrm{v}$ ACN:40-20 v/v GAA between 7 and 10 minutes followed by $80 \%$ ACN:20\% GAA between 10 and 15 minutes.

\section{Method Validation}

The optimized method for the simultaneous estimation of curcumin and quercetin has been validated as per ICH Q2 (R1) guidelines ${ }^{33}$ for evaluating system suitability, specificity, precision, accuracy, linearity, range, LOD, LOQ, and robustness.

System suitability. System suitability parameters such as theoretical plate number, tailing factor, resolution between curcumin and quercetin peaks, and height equivalent to theoretical plate (HETP) for both drugs were calculated by injecting a blank mobile phase followed by six replicates of $250 \mathrm{ng}$ curcumin-quercetin mixture onto the HPLC system. ${ }^{34}$

Linearity and range. The range was selected for both the drugs starting by injecting the minimum concentration of both the drugs $\left(10 \mathrm{ng} \mathrm{mL}^{-1}\right)$. After selection of the range, different dilutions of curcumin $(\mathrm{C} 1, \mathrm{C} 2, \mathrm{C} 3$, $\mathrm{C} 4$, and C5) and quercetin (Q6, Q7, Q8, Q9, and Q10) were injected onto the HPLC system. Five injections of each concentration $(\mathrm{C} 1, \mathrm{C} 2, \mathrm{C} 3, \mathrm{C} 4$, and $\mathrm{C} 5)$ were plotted on a curcumin calibration curve. Similarly, 5 injections of each concentration (Q6, Q7, Q8, Q9, and Q10) were plotted on a quercetin calibration curve. The slope, intercept, and correlation coefficient of the calibration curves (peak area vs concentration) were determined to ensure linearity of the analytical method. ${ }^{34}$

Accuracy study. An accuracy study was performed based on absolute recovery at three levels, ie, 80, 100, and $120 \%$ of the mid concentration of the calibration curve (i.e., $150 \mathrm{ng} \mathrm{mL}^{-1}$ for curcumin and $1.2 \mu \mathrm{g} \mathrm{mL}^{-1}$ for quercetin). These levels were labeled as lower 
quantified concentration (LQC), medium quantified concentration (MQC), and high quantified concentration (HQC). In the case of curcumin, LQC, MQC, and HQC were 120,150 , and $180 \mathrm{ng}$. In the case of quercetin, LQC, MQC, and HQC were 960, 1200, and $1440 \mathrm{ng}$. The accuracy was expressed in terms of percentage recovery of curcumin and quercetin from the solution. The study was carried out six times, and mean data were recorded. Percentage absolute recovery was calculated by following equation: ${ }^{34}$

$$
\begin{aligned}
\text { Absolute recovery }(\%)= & \frac{\text { Actual concentration recovered }}{\text { Theoritical concentration }} \\
& * 100
\end{aligned}
$$

Precision studies. A precision study was carried out in two steps, namely, repeatability and intermediate precision. Repeatability was carried out by injecting six injections at three levels, i.e., LQC, MQC, and HQC, simultaneously within a day, whereas intermediate precision was carried out in two steps. In step 1, interday study was carried out by injecting LQC, MQC, and HQC samples six times each for three different days. In step 2, interanalyst study was carried out by injecting LQC, MQC, and HQC samples six times each prepared by three different analysts under the same experimental condition. ${ }^{34}$ The mean data were recorded, and percentage relative standard deviation was calculated.

Determination of LOD and LOQ. LOD and LOQ for curcumin and quercetin were calculated from the linear regression equation based on the standard deviation of the intercept and the slope using equations (2a) and (2b), respectively, ${ }^{34}$

$$
\begin{aligned}
& \text { LOD }=\frac{3.3 Q}{S} \\
& \text { LOQ }=\frac{10 Q}{S}
\end{aligned}
$$

where $Q$ is the standard deviation of the intercept and $S$ is the slope of the calibration curve.

Robustness. Deliberate minute variations in the chromatographic conditions such as the flow rate and wavelength have been made. These variations were also assessed for resolution between curcumin and quercetin peaks, number of theoretical plates, and tailing factor. ${ }^{34}$

Method specificity and applicability. Quantification of quercetin and curcumin in herbal extracts and marketed formulations. For evaluating the applicability of the developed method, each extract of quercetin (onion, pudina, ajwain, amla, chebula, ginger, green tea, nutmeg, pepper, pomegranate, and tulsi) $(100 \mathrm{mg})$ was weighed and dissolved in $10 \mathrm{~mL}$ of ACN taken in a test tube. Similarly, each marketed formulation of Curcuma longa extract containing curcumin was dissolved in ACN to produce the final concentration of $5 \mu \mathrm{g} \mathrm{mL} \mathrm{m}^{-1}$. Each sample was vortexed (CM 101 CYCLO MIXER, REMI, India) for 5 minutes. After that, it was kept as such for 24 hours with intermediate vortexing and sonication. After 24 hours, each sample was centrifuged (REMI CM-12 PLUS, India) for 20 minutes at $9262 g$ for the removal of the undissolved powder from saturated solutions. The supernatant from each sample was removed and filtered through a syringe filter and then injected onto the HPLC system to analyze the peak of curcumin and quercetin present in extracts.

Drug loading in SNEDDS. In addition to this, drug loading of developed SNEDDS formulation loaded with curcumin and quercetin was also evaluated using this simultaneous method.

\section{Formulation of SNEDDS}

For preparing liquid SNEDDSs of curcumin and quercetin, $1 \mathrm{~mL}$ of the isotropic mixture was prepared using Lafrafill M1944CS, Capmul MCM, Transcutol P, Labrasol, and Tween 80. Curcumin and quercetin (each $5 \mathrm{mg}$ ) were added and dissolved in the isotropic mixture. The isotropic mixture was vortexed (CM 101 CYCLO MIXER, REMI, India) for 5 minutes. It was sonicated (Labman-ROHS, China) for 5 minutes and then centrifuged (REMI CM-12 PLUS, India) for 20 minutes at $9262 \mathrm{~g}$ for the removal of the undissolved drug from the saturated solution. The supernatant from the isotropic mixture was removed and diluted to $500 \mathrm{~mL}$ with distilled water kept on a magnetic stirrer. The sample with a concentration of $10 \mu \mathrm{g} \mathrm{mL}^{-1}$ was filtered through a syringe filter and then injected onto the HPLC system to analyze the peak of curcumin and quercetin in SNEDDS formulation. The standard was prepared by dissolving $10 \mathrm{mg}$ of each drug in $100 \mathrm{~mL}$ ACN. From this stock solution $\left(100 \mu \mathrm{g} \mathrm{mL}^{-1}\right), 1 \mathrm{~mL}$ was taken and diluted to $10 \mathrm{~mL}$ to produce the standard with a concentration of $10 \mu \mathrm{g} \mathrm{mL}^{-1}$ for curcumin and quercetin each. This solution was further injected onto the HPLC system to analyze the peak of standard curcumin and quercetin. The percentage drug loading was evaluated using the following equation: ${ }^{35,36}$

$$
\% \text { Drug loading }=\frac{\text { Concentration of formulation }}{\text { Concentration of standard }} * 100
$$

\section{Specificity studies}

The effect of excipients used in the formulation was determined. The specificity study was carried out in order to check any possible interaction of excipients 




FIG. 1. Isobestic point of curcumin and quercetin.

Table 1. Optimized gradient elution for simultaneous estimation of curcumin and quercetin.

\begin{tabular}{cc} 
Time $(\min )$ & $(\% A C N)$ v/v \\
\hline 0.01 & 40 \\
5 & 60 \\
8 & 80 \\
15 & Stop \\
\hline
\end{tabular}

with the drugs. Each solubilizer and excipient (Tween 80, Labrafil M1944CS, Transcutol P, Labrasol, and Capmul MCM) to be used for the formulation of SNEDDS $(100 \mu \mathrm{L})$ was diluted with either ethanol or hexane depending upon the solubility and then injected on the HPLC system. The specificity of the proposed HPLC method for the determination of curcumin and quercetin has been established by injecting the mobile phase and placebo SNEDDS solution into the HPLC system.

\section{Results and Discussion}

Selection of UV Wavelength

Curcumin has an absorption maxima around $425 \mathrm{~nm},{ }^{37}$ and quercetin has absorption maxima at $370 \mathrm{~nm} .{ }^{38}$ From the UV results, the absorption maxima of quercetin were found to be around $365 \mathrm{~nm}$, and the absorption maxima of curcumin were found to be $420 \mathrm{~nm}$. The isobestic point of both the drugs in combination is found to be at $395 \mathrm{~nm}$ as depicted in Fig. 1. The method development and validation for both the drugs were done at $395 \mathrm{~nm}$.

\section{Method Development and Optimization}

In trial 1 , retention times for quercetin and curcumin were found to be 3.4 and 7.2 minutes, respectively.



FIG. 2. Optimized HPLC chromatogram of curcumin and quercetin.

However, the peaks showed broadening and shouldering. Hence, the method was not considered for further validation. In trial 2, the method was rejected since the peak of quercetin was not sharp due to broadening. In trial 3, retention time for quercetin and curcumin was 4.1 and 14 minutes, respectively, which was too long. So this trial was also rejected. Trial 4 was rejected since the peak shape of quercetin was not good due to observed fronting and curcumin was not eluted until 20 minutes. Trial 5 (Table 1) was chosen for further method validation as the peaks were sharp with no tailing or fronting. Furthermore, the theoretical plate value for curcumin and quercetin in this method was 56,626 and 3297, which was above 2000. HETP for curcumin and quercetin was 2.64 and 45.49 , respectively. The tailing factor for both the drugs was less than 2 . The peaks of both curcumin and quercetin for the developed method are given in Fig. 2.

\section{System Suitability}

The obtained results of hexaplicate injections for both the drugs exhibited that the limits tested were within the acceptable range. Curcumin and quercetin were repeatedly retained and well separated at 6.1 and 11.9 minutes, stating very good resolution between both peaks with relative standard deviation percent (\% RSD) of the recorded retention times $<0.2$ to specify excellent repeatability of replicate injections on the integral HPLC system used; the tailing factor for both curcumin and quercetin peaks never exceeded 2 in all peaks, demonstrating good peak regularity (acceptance limit is $<2$ ); and the number of theoretical plates were always $>2000$ in all chromatographic runs, ensuring good column efficacy throughout the developed separation process. It is important to note that very small two peaks at 11.3 and 11.5 minutes were also observed along with the peak of curcumin belonging to its other forms, namely, curcuminoid A and B, however, with very low amount. Results are presented in Table 2. 
Table 2. System suitability results for curcumin and quercetin.

\begin{tabular}{lccc} 
& \multicolumn{2}{c}{ Value } & \\
\cline { 2 - 3 } & Curcumin & Quercetin & \\
\hline HETP & 2.649 & 45.218 & Depends on theoretical plate \\
Theoretical plate & $56,626.8$ & 3317 & $>2000$ \\
Theoretical plate/meter & 377,512 & 22115 & $>20,000$ \\
Tailing factor (symmetry factor) & 1.128 & 0.918 & $<2$ \\
Peak purity index & 0.999 & 0.837 & $>0.5$ \\
\hline
\end{tabular}

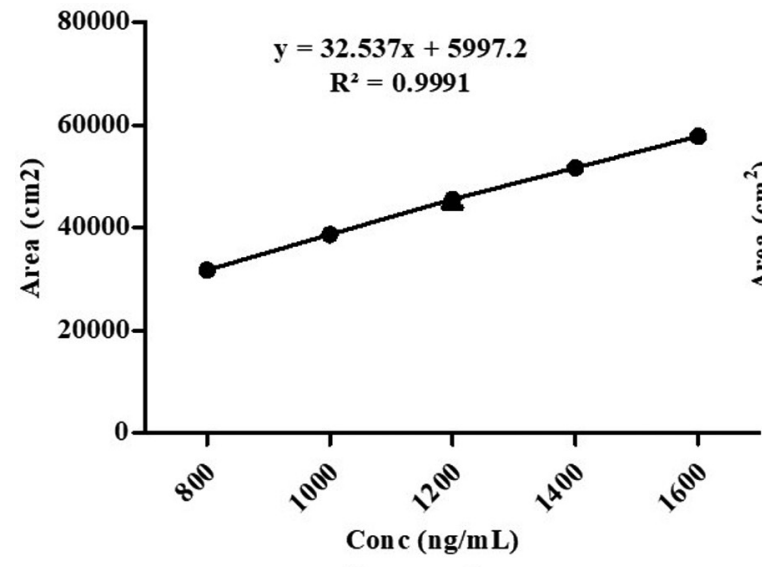

a. Quercetin

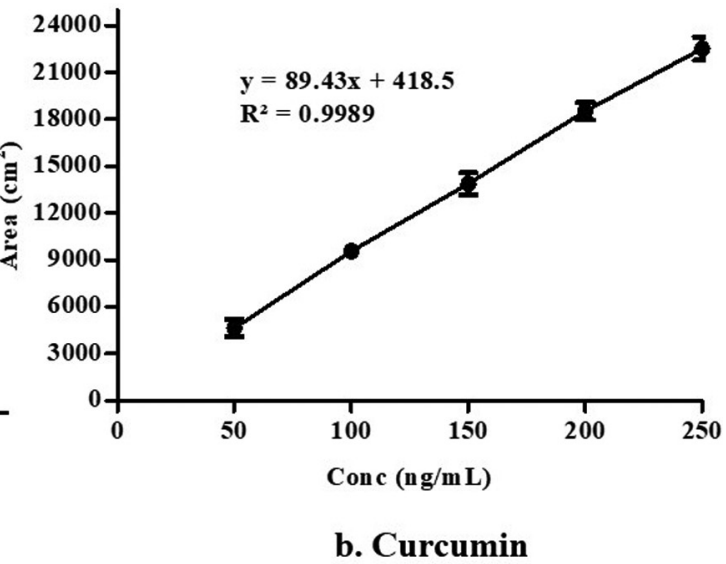

b. Curcumin

FIG. 3. Calibration curve of (A) quercetin and (B) curcumin.

Linearity and Range

The calibration curves created for both quercetin (Fig. 3A) and curcumin (Fig. 3B) were linear in the specified ranges of $800-1600 \mathrm{ng} \mathrm{mL}^{-1}$ for quercetin and $50-250 \mathrm{ng} \mathrm{mL}^{-1}$ for curcumin. The correlation coefficient $\left(r^{2}\right)$ for curcumin was noted as 0.9989 with the linear regression equation $Y=89.43 x+418.5$. Similarly, for quercetin, the curve $r^{2}$ was noted as 0.9991 with a linear regression equation $Y=32.537 x+5997.2$. The rationale for developing linearity at different ranges for curcumin and quercetin was based on their linearity. A good regression coefficient $\left(r^{2}\right)$ was observed for curcumin in the range of $50-250 \mathrm{ng} \mathrm{mL}^{-1}$, but for quercetin, it was not observed in this range. Hence, a higher value of range was selected for quercetin.

\section{Accuracy}

Accuracy of the developed HPLC analytical method was assessed by calculating percentage recovery that was found to be in the range of $95-105 \%$ with RSD less than $2 \%$ indicating the accuracy of method for both the drugs (Table 3).

\section{Precision}

The results of interday and intraday studies were carried out by injecting LQC, MQC, and HQC. The peak areas separately found for curcumin and quercetin were repeatable and precise over three successive days. The results for both intraday and interday findings ensure the high repeatability and precision of the developed method where all data were expressed in \% RSD, which was always under the acceptance limit (\% RSD <2). Results for intra- and interday precision for curcumin and quercetin are given in Table 4.

\section{LOD and LOQ}

The LOD was 11.96 and $32.94 \mathrm{ng} \mathrm{mL}^{-1}$ for curcumin and quercetin, respectively, whereas, the LOQ was 35.6 and $99.76 \mathrm{ng} \mathrm{mL}^{-1}$ for curcumin and quercetin, respectively.

\section{Robustness}

There were no significant changes noticed upon varying some of the parameters of the chromatographic conditions confirming that the method is robust to small deliberate changes applied in terms of the flow rate and wavelength. In all cases, curcumin and quercetin peaks were symmetric (tailing factor $<2$ ) and were well separated (resolution $>2$ ). The $\%$ RSD of curcumin and quercetin retention times were $<1$ confirming the robustness of the developed analytical method to small changes. Results for robustness are presented in Table 5. 
Table 3. Accuracy study results for curcumin and quercetin.

\begin{tabular}{|c|c|c|c|c|c|}
\hline Levels & $\begin{array}{l}\text { Theoritical concentration } \\
\text { of standard solution }(\mathrm{ng} / \mathrm{mL})\end{array}$ & $\begin{array}{l}\text { Acutal concentration recovered } \\
\text { from mobile phase }(\mathrm{ng} / \mathrm{mL}) \\
(\text { mean } \pm S D)(N=6)\end{array}$ & $R S D(\%)$ & $\begin{array}{l}\text { Recovery (\%) } \\
(\text { mean } \pm S D)\end{array}$ & $R S D(\%)$ \\
\hline \multicolumn{6}{|l|}{ Curcumin } \\
\hline LQC & 120 & $116.72 \pm 1.17$ & 1.094 & $97.26 \pm 0.95$ & 0.98 \\
\hline MQC & 150 & $148.94 \pm 1.94$ & 1.30 & $99.29 \pm 1.32$ & 1.33 \\
\hline HQC & 180 & $189.2 \pm 1.42$ & 0.75 & $105.49 \pm 0.81$ & 0.77 \\
\hline \multicolumn{6}{|l|}{ Quercetin } \\
\hline LQC & 960 & $920 \pm 4.68$ & 1.34 & $96.5 \pm 0.34$ & 0.35 \\
\hline MQC & 1200 & $1217 \pm 3.40$ & 0.28 & $101.4 \pm 0.78$ & 0.77 \\
\hline HQC & 1440 & $1503 \pm 1.29$ & 0.085 & $104.4 \pm 0.24$ & 0.23 \\
\hline
\end{tabular}

$\mathrm{SD}$, standard deviation; RSD, relative standard deviation.

Table 4. Precision study results of curcumin and quercetin.

\begin{tabular}{|c|c|c|c|c|}
\hline Parameters & Levels & Conc $\left(n g m L^{-1}\right)$ & Mean area $\pm S D(N=6)\left(\mathrm{cm}^{2}\right)$ & RSD (\%) \\
\hline \multicolumn{5}{|l|}{ Curcumin } \\
\hline \multirow[t]{3}{*}{ Repeatability (intraday precision) } & LQC & 120 & $10896.00 \pm 183.42$ & 1.68 \\
\hline & MQC & 150 & $13728.67 \pm 195.81$ & 1.42 \\
\hline & HQC & 180 & $17314.00 \pm 224.32$ & 1.29 \\
\hline \multirow[t]{3}{*}{ Interanalyst } & LQC & $120(\mathrm{~A} 1, \mathrm{~A} 2, \mathrm{~A} 3)$ & $10805.00 \pm 81.43$ & 0.75 \\
\hline & MQC & $150(\mathrm{~A} 1, \mathrm{~A} 2, \mathrm{~A} 3)$ & $13427.67 \pm 265.12$ & 1.97 \\
\hline & HQC & $180(\mathrm{~A} 1, \mathrm{~A} 2, \mathrm{~A} 3)$ & $17620.00 \pm 305.50$ & 1.73 \\
\hline \multicolumn{5}{|l|}{ Intermediate precision (interday) } \\
\hline Day 1, Day 2, Day 3 & LQC & 120 & $10777.67 \pm 106.03$ & 0.98 \\
\hline Day 1, Day 2, Day 3 & MQC & 150 & $13697.67 \pm 176.46$ & 1.28 \\
\hline Day 1 , Day 2, Day 3 & HQC & 180 & $17409.67 \pm 129.47$ & 0.74 \\
\hline \multicolumn{5}{|l|}{ Mean of all precision studies for curcumin } \\
\hline \multirow[t]{3}{*}{ Intraday, Interanalyst, and Interday precision } & LQC & 120 & $32,478.36 \pm 456.22$ & 1.40 \\
\hline & MQC & 150 & $40852.45 \pm 667.18$ & 1.63 \\
\hline & $\mathrm{HQC}$ & 180 & $52343.22 \pm 967.19$ & 1.85 \\
\hline \multicolumn{5}{|l|}{ Quercetin } \\
\hline \multirow[t]{3}{*}{ Repeatability (Intraday) precision) } & LQC & 960 & $35418.67 \pm 303.21$ & 0.85 \\
\hline & MQC & 1200 & $45437.33 \pm 231.59$ & 0.50 \\
\hline & HQC & 1440 & $55539.15 \pm 258.12$ & 0.46 \\
\hline \multirow[t]{3}{*}{ Interanalyst } & LQC & $960(\mathrm{~A} 1, \mathrm{~A} 2, \mathrm{~A} 3)$ & $35108.33 \pm 581.24$ & 1.65 \\
\hline & MQC & 1200(A1, A2, A3) & $45275.33 \pm 279.14$ & 0.61 \\
\hline & HQC & $1440(\mathrm{~A} 1, \mathrm{~A} 2, \mathrm{~A} 3)$ & $55397.22 \pm 559.32$ & 1.00 \\
\hline \multicolumn{5}{|l|}{ Intermediate precision (interday) } \\
\hline Day 1, Day 2, Day 3 & LQC & 960 & $34880.67 \pm 468.82$ & 1.34 \\
\hline Day 1, Day 2, Day 3 & MQC & 1200 & $45435.33 \pm 341.50$ & 0.75 \\
\hline Day 1, Day 2, Day 3 & HQC & 1440 & $55816.67 \pm 129.67$ & 0.23 \\
\hline \multicolumn{5}{|l|}{ Mean of all precision studies for quercetin } \\
\hline \multirow[t]{3}{*}{ Intraday, Interanalyst, and Interday precision } & LQC & 960 & $105,407.67 \pm 1233.34$ & 1.17 \\
\hline & MQC & 1200 & $136,147.99 \pm 1866.87$ & 1.37 \\
\hline & HQC & 1440 & $166,753.04 \pm 2465.15$ & 1.49 \\
\hline
\end{tabular}

$\mathrm{N}$, response of number of replicates of study conducted; SD, standard deviation; RSD, relative standard deviation; A, analyst.

\section{Method Applicability}

The applicability of the method has been confirmed where the optimized conditions were applied to detect the presence of curcumin and quercetin in different quercetin extracts and marketed formulations of curcumin. The representative chromatograms of quercetin peaks in extracts and curcumin peaks in marketed formulations are shown in Supplementary Figs. S1 and S2, respectively. Furthermore, the amount of curcumin and quercetin present in these extracts has been calculated and is important to note that the concentrations of curcumin present in the extracts of Allium cepa (onion) and Mentha arvensis (pudina) are below the LOQ of curcumin. These extracts include. The applicability of the developed method was also checked in our formulated SNEDDS. The respective peaks of curcumin and quercetin from the SNEDDS formulation are presented in Supplementary Fig. S3. The method was further used to calculate drug loading.

The drug loading in the case of quercetin was found to be $96 \pm 1.21 \%$, whereas in the case of curcumin, it 
Table 5. Robustness study of curcumin and querectin.

\begin{tabular}{|c|c|c|c|c|c|c|}
\hline Variables & Value & Conc. $(n g / m L)$ & Mean area $\pm S D(N=5)\left(\mathrm{cm}^{2}\right)$ & RSD (\%) & Mean $R_{t}(\min ) \pm S D(N=5)$ & RSD (\%) \\
\hline Curcumin & & & & & & \\
\hline \multirow{3}{*}{ Flow rate $\left(\mathrm{mL} \min ^{-1}\right)$} & 0.8 & 150 & $18,585.2 \pm 328.33$ & 1.76 & $13.54 \pm 0.07$ & 0.54 \\
\hline & 1 & 150 & $13,715.4 \pm 209.47$ & 1.52 & $12.04 \pm 0.01$ & 0.15 \\
\hline & 1.2 & 150 & $11,452.4 \pm 155.69$ & 1.35 & $10.74 \pm 0.03$ & 0.28 \\
\hline \multirow[t]{3}{*}{ Wavelength (nm) } & 393 & 150 & $12,895.2 \pm 240.29$ & 1.86 & $12.02 \pm 0.01$ & 0.14 \\
\hline & 395 & 150 & $13,523.6 \pm 232.14$ & 1.71 & $12.02 \pm 0.01$ & 0.15 \\
\hline & 297 & 150 & $14,074.2 \pm 194.40$ & 1.38 & $12.0 \pm 0.02$ & 0.21 \\
\hline \multicolumn{7}{|l|}{ Quercetin } \\
\hline \multirow{3}{*}{ Flow rate $\left(\mathrm{mL} \min ^{-1}\right)$} & 0.8 & 1200 & $54,326 \pm 114.46$ & 0.21 & $7.22 \pm 0.09$ & 1.28 \\
\hline & 1 & 1200 & $45,494.6 \pm 206.02$ & 0.45 & $5.88 \pm 0.06$ & 1.02 \\
\hline & 1.2 & 1200 & $37,148.8 \pm 258.07$ & 0.69 & $4.91 \pm 0.04$ & 0.85 \\
\hline \multirow[t]{3}{*}{ Wavelength (nm) } & 393 & 1200 & $51,185 \pm 175.64$ & 0.34 & $5.88 \pm 0.06$ & 1.062 \\
\hline & 395 & 1200 & $45,494.6 \pm 206.02$ & 0.45 & $5.88 \pm 0.06$ & 1.02 \\
\hline & 297 & 1200 & $39,624.8 \pm 335.48$ & 0.84 & $5.88 \pm 0.06$ & 1.02 \\
\hline
\end{tabular}

Table 6. Amount of curcumin in different extracts.

\begin{tabular}{lcc}
\hline 1. & Trachyspermum ammi (Ajwain) & $45 \pm 1.46$ \\
2. & Phyllanthus embelica (Amla) & $280 \pm 3.39$ \\
3. & Terminalia chebula (Myrobalan) & $364 \pm 1.87$ \\
4. & Zingiber officinale (Ginger) & $60 \pm 3.56$ \\
5. & Camellia sinensis (Green tea) & $1100 \pm 19.07$ \\
6. & Myristica fragrans (Nutmeg) & $2550 \pm 17.65$ \\
7. & Piper longum (Pepper) & $92 \pm 3.87$ \\
8. & Punica granatum (Pommegranate) & $142 \pm 1.76$ \\
9. & Ocimum tenuiflorum (Tulsi) & $390 \pm 3.36$ \\
\hline
\end{tabular}

was found to be $99.9 \pm 1.01 \%$ when loaded into LSNEDDS.

\section{Specificity}

The absence of excipient peaks used to formulate SNEDDS in the chromatogram of curcumin and quercetin indicated the selectivity of the developed method. The chromatograms for the blank samples of these excipients are given in Supplementary Fig. S4.

\section{Conclusion}

In this study, an attempt has been made to develop an RP-HPLC method for simultaneous estimation of curcumin and quercetin. The method was successfully developed and complied with all method validation parameters such as linearity and range, precision, and accuracy. In addition to this, the method was successfully applied to quantify the presence of curcumin and quercetin in various extracts, marketed formulations, and SNEDDSs, which indicated toward selectivity and sensitivity of the developed method. The obtained positive outcomes of this study indicated that the developed method can be further explored to quantify these drugs in biological samples during the preclinical or clinical studies.

Declaring Adherence to Ethical Guidelines: The study presented in the manuscript does not contain any
Table 7. Amount of quercetin in different extracts.

\begin{tabular}{lcc} 
S.No. & Formulation & Amount $\left(\mathrm{ng} \mathrm{m}^{-1}\right)$ \\
\hline 1. & Turmeric Formula & $3220 \pm 32.33$ \\
2. & Himalaya Haridra & $180 \pm 12.76$ \\
3. & Haldi immunity enhancer & $106 \pm 5.98$ \\
4. & Curpin & $5360 \pm 18.08$ \\
5. & Haridra Khand & $130 \pm 20.22$ \\
6. & Dabur Haridra Khand & $490 \pm 17.43$ \\
\hline
\end{tabular}

plagiarized contents. It has been submitted only to this journal. There are no animals/humans involved in this study.

Authors' Disclosure Statements: Declared none.

Authorship Contribution Statement: All authors have read and approved the manuscript. R.K. contributed to methodology, data curation, and writing-original draft. S.K.S. contributed to conceptualization, validation, supervision, and writing-review and editing. B.K., M.G., and S.W. involved in supervision and writingreview and editing. S.G., P.P., D.K., L.M.S.K., U.V.B., M.S., H.K.S., and V.K. contributed to methodology. K.D. involved in review and editing.

Funding Statement: This work did not receive any grant.

\section{Acknowledgments}

The authors are thankful to The Himalaya Drugs Company, Bengaluru, India, and Vasu Research Centre, Vadodara, Gujarat, India, for providing gift samples of extracts.

\section{References}

1. Chandran B, Goel A. A randomized, pilot study to assess the efficacy and safety of curcumin in patients with active rheumatoid arthritis. Phytother Res 2012; 26(11): 1719-1725. DOI: 10.1002/ptr.4639

2. Kowluru RA, Kanwar M. Effects of curcumin on retinal oxidative stress and inflammation in diabetes. Nutr Metab (Lond) 2007; 4(1): 1-8. DOI: $10.1186 / 1743-7075-4-8$ 
3. Kim J-H, Kang M-J, Choi H-N, et al. Quercetin attenuates fasting and postprandial hyperglycemia in animal models of diabetes mellitus. Nutr Res Pract 2011; 5(2): 107-111. DOI: 10.4162/nrp.2011.5.2.107

4. Egert S, Bosy-Westphal A, Seiberl J, et al. Quercetin reduces systolic blood pressure and plasma oxidised low-density lipoprotein concentrations in overweight subjects with a high-cardiovascular disease risk phenotype: A double-blinded, placebo-controlled cross-over study. Br J Nutr 2009; 102(7): 1065-1074. DOI: 10.1017/S0007114509359127

5. Campbell MS, Ouyang A, Krishnakumar I, et al. Influence of enhanced bioavailable curcumin on obesity-associated cardiovascular disease risk factors and arterial function: A double-blinded, randomized, controlled trial. Nutrition 2019; 62: 135-139. DOI: 10.1016/j.nut.2019.01.002

6. Khursheed R, Singh SK, Wadhwa $S$, et al. Treatment strategies against diabetes: Success so far and challenges ahead. Eur J Pharmacol 2019; 862: 172625. DOI: 10.1016/j.ejphar.2019.172625

7. Khan M, Ali M, Shah W, et al. Curcumin-loaded self-emulsifying drug delivery system (cu-SEDDS): a promising approach for the control of primary pathogen and secondary bacterial infections in cutaneous leishmaniasis. Appl Microbiol Biotechnol 2019; 103(18): 7481-7490. DOI: 10.1007/ s00253-019-09990-x

8. Rivera-Mancía S, Trujillo J, Chaverri JP. Utility of curcumin for the treatment of diabetes mellitus: Evidence from preclinical and clinical studies. J Nutr Intermed Metab 2018; 14: 29-41. DOI: 10.1016/j.jnim.2018.05.001

9. Hoda ME, Pierre SH. The antidiabetic potential of quercetin: Underlying mechanisms. Curr Med Chem 2017; 24(4): 355-364.

10. Khursheed R, Singh SK, Wadhwa S, et al. Enhancing the potential preclinical and clinical benefits of quercetin through novel drug delivery systems. Drug Discov Today 2019.

11. Liu Z, Cui C, Xu P, et al. Curcumin activates AMPK pathway and regulates lipid metabolism in rats following prolonged clozapine exposure. Front Neurosci 2017; 11: 558. DOI: 10.3389/fnins.2017.00558

12. Ahn J, Lee $H, K i m ~ S$, et al. The anti-obesity effect of quercetin is mediated by the AMPK and MAPK signaling pathways. Biochem Biophys Res Commun 2008; 373(4): 545-549. DOI: 10.1016/j.bbrc.2008.06.077

13. Sun L, Li E, Wang F, et al. Quercetin increases macrophage cholesterol efflux to inhibit foam cell formation through activating PPAR $\gamma-A B C A 1$ pathway. Int J Clin Exp Pathol 2015; 8(9): 10854-10860.

14. Pisonero-Vaquero S, Martínez-Ferreras Á, García-Mediavilla MV, et al. Quercetin ameliorates dysregulation of lipid metabolism genes via the $\mathrm{PI} 3 \mathrm{~K} / \mathrm{AKT}$ pathway in a diet-induced mouse model of nonalcoholic fatty liver disease. Mol Nutr Food Res 2015; 59(5): 879-893.

15. Hussain A, Al-Rasheed M, Manogaran $P$, et al. Curcumin induces apoptosis via inhibition of $\mathrm{PI} 3^{\prime}$-kinase/AKT pathway in acute T cell leukemias. Apoptosis 2006; 11(2): 245-254. DOI: 10.1007/s10495-006-3392-3

16. Muthian G, Bright JJ. Quercetin, a flavonoid phytoestrogen, ameliorates experimental allergic encephalomyelitis by blocking IL-12 signaling through JAK-STAT pathway in T lymphocyte. J Clin Immunol 2004; 24(5): 542-552. DOI: 10.1023/B:JOCI.0000040925.55682.a5

17. Fonseca-Santos B, Gremião MPD, Chorilli M. A simple reversed phase high-performance liquid chromatography (HPLC) method for determination of in situ gelling curcumin-loaded liquid crystals in in vitro performance tests. Arab J Chem 2017; 10(7): 1029-1037. DOI: 10.1016/ j.arabjc.2016.01.014

18. Singh RSP, Das U, Dimmock JR, et al. A general HPLC-UV method for the quantitative determination of curcumin analogues containing the 1,5 diaryl-3-oxo-1,4-pentadienyl pharmacophore in rat biomatrices. J Chromatogr B 2010; 878(28): 2796-2802. DOI: 10.1016/j.jchromb.2010.08.034

19. Rahimi M, Bahar S, Heydari R, et al. Determination of quercetin using a molecularly imprinted polymer as solid-phase microextraction sorbent and high-performance liquid chromatography. Microchem J 2019; 148 433-441. DOI: 10.1016/j.microc.2019.05.032

20. Pilařová $V$, Plachká $K$, Chrenková $L$, et al. Simultaneous determination of quercetin and its metabolites in rat plasma by using ultra-high performance liquid chromatography tandem mass spectrometry. Talanta 2018; 185: 71-79. DOI: 10.1016/j.talanta.2018.03.033

21. Kim DW, Yousaf AM, Li DX, et al. Development of RP-HPLC method for simultaneous determination of docetaxel and curcumin in rat plasma:
Validation and stability. Asian J Pharmaceut Sci 2017; 12(1): 105-113. DOI: 10.1016/j.ajps.2016.08.002

22. Kharat S, Namdeo A, Mehta P. Development and validation of HPTLC method for simultaneous estimation of curcumin and galangin in polyherbal capsule dosage form. J Taibah Univ Sci 2017; 11(5): 775-781. DOI: 10.1016/j.jtusci.2016.10.004

23. Kumar S, Lather V, Pandita D. Stability indicating simplified HPLC method for simultaneous analysis of resveratrol and quercetin in nanoparticles and human plasma. Food Chem 2016; 197: 959-964. DOI: 10.1016/j.foodchem.2015.11.078

24. Seo $M-J$, Lee $Y-J$, Hwang $J-H$, et al. The inhibitory effects of quercetin on obesity and obesity-induced inflammation by regulation of MAPK signaling. J Nutr Biochem 2015; 26(11): 1308-1316. DOI: 10.1016/ j.jnutbio.2015.06.005

25. Weisberg SP, Leibel R, Tortoriello DV. Dietary curcumin significantly improves obesity-associated inflammation and diabetes in mouse models of diabesity. Endocrinology 2008; 149(7): 3549-3558. DOI: 10.1210/en.2008-0262

26. Reda FM, El-Saadony MT, Elnesr SS, et al. Effect of dietary supplementation of biological curcumin nanoparticles on growth and carcass traits, antioxidant status, immunity and caecal microbiota of Japanese quails. Animals 2020; 10(5): 754. DOI: 10.3390/ani10050754

27. Cruz-Correa M, Shoskes DA, Sanchez $P$, et al. Combination treatment with curcumin and quercetin of adenomas in familial adenomatous polyposis. Clin Gastroenterol Hepatol 2006; 4(8): 1035-1038. DOI: 10.1016/ j.cgh.2006.03.020

28. Amazon.com: Turmeric Curcumin Bromelain Quercetin Bioperine. Occasional Joint Discomfort Relief Supplement. Antioxidant Properties for Immune, Heart, Digestive Health. Supports Balanced Inflammatory Response. Health \& Personal Care. Accessed August 2, 2021.

29. Yu W, Wen D, Cai D, et al. Simultaneous determination of curcumin, tetrahydrocurcumin, quercetin, and paeoniflorin by UHPLC-MS/MS in rat plasma and its application to a pharmacokinetic study. J Pharmaceut Biomed Anal 2019; 172: 58-66. DOI: 10.1016/j.jpba.2019.04.033

30. Ang LF, Yam MF, Fung YTT, et al. HPLC method for simultaneous quantitative detection of quercetin and curcuminoids in traditional Chinese medicines. J Pharmacopunct 2014; 17(4): 36-49. DOI: 10.3831/ KPI.2014.17.035

31. Baghel U, Nagar A, Pannu M, et al. HPLC and HPTLC methods for simultaneous estimation of quercetin and curcumin in polyherbal formulation. $J$ Pharmaceut Sci 2017; 79. DOI: 10.4172/pharmaceutical-sciences.1000217

32. Ang L, Yam M, Fung Y, et al. HPLC method for simultaneous quantitative detection of quercetin and curcuminoids in traditional Chinese medicines. J Pharmacopunct 2014; 17: 36-49. DOI: 10.3831/KPI.2014.17.035

33. Mangla B, Beg S, Alam O, et al. Systematic development and validation of RP-HPLC method for simultaneous estimation of tamoxifen and sulphoraphane with specific application for nanolipidic formulations. Arab J Chem 2020; 13(11): 7909-7920. DOI: 10.1016/j.arabjc.2020.09.022

34. Kumar R, Kumar R, Khursheed R, et al. Development and validation of RP-HPLC method for estimation of fisetin in rat plasma. S Afr J Bot 2020. DOI: $10.1016 /$ j.sajb.2020.05.010

35. Khursheed R, Singh SK, Wadhwa S, et al. Exploring role of probiotics and Ganoderma lucidum extract powder as solid carriers to solidify liquid self-nanoemulsifying delivery systems loaded with curcumin. Carbohydr Polym 2020; 250: 116996. DOI: 10.1016/j.carbpol.2020.116996

36. Kumar R, Khursheed R, Kumar R, et al. Self-nanoemulsifying drug delivery system of fisetin: Formulation, optimization, characterization and cytotoxicity assessment. J Drug Deliv Sci Technol 2019; 54: 101252. DOI: 10.1016/j.jddst.2019.101252

37. Rapalli VK, Kaul V, Gorantla S, et al. UV spectrophotometric method for characterization of curcumin loaded nanostructured lipid nanocarriers in simulated conditions: Method development, in-vitro and ex-vivo applications in topical delivery. Spectrochim Acta Part A: Mol Biomol SpectrosC 2020; 224: 117392-DOI: 10.1016/j.saa.2019.117392

38. He Y, He Z, He F, et al. Determination of quercetin, plumbagin and total flavonoids. Phcog Mag 2012; 8(32): 263-267. DOI: 10.4103/09731296.103649 


\section{Author Query}

AQ1: Please provide structured abstract as per the style.

AQ2: Please note that the Table $6 a$ and Table $6 \mathrm{~b}$ have been renumbered as Table 6 and Table 7, respectively.

AQ3: Please reword the sentence beginning with "These extracts include..." so that your meaning will be clear to the reader.

AQ4: Please provide volume number and page range for Refs. 10 and 34.

AQ5: Please provide URL or more details in Ref. 28.

AQ6: Please provide page range in Ref. 31.

AQ7: References 30 and 32 contain identical information. Please check and provide the correct reference or delete the duplicate reference. If the duplicate is deleted, renumber the reference list as needed and update all citations in the text. 